\title{
gẹ́ographie economie société
}

\section{Les marchés immobiliers : acteurs, institutions et territoires}

\author{
Thierry Theurillat ${ }^{\mathrm{a}^{*}}$, Patrick Rérat ${ }^{\mathrm{a}, \mathrm{b}}$ et Olivier Crevoisier ${ }^{\mathrm{a}}$ \\ ${ }^{a}$ Groupe de recherche en Economie territoriale (GRET) \\ Institut de Sociologie, Université de Neuchâtel, Suisse \\ ${ }^{b}$ Institut de géographie et durabilité \\ Université de Lausanne, Suisse
}

\section{Résumé}

Par la mise en évidence des acteurs, des processus et des institutions et par conséquent de la diversité ainsi que des contingences des marchés immobiliers, la littérature actuelle sur l'immobilier insiste sur les nombreux liens et interdépendances entre l'immobilier, le foncier, l'urbanisme et les politiques urbaines ou encore avec le système financier. Toutefois, l'essentiel de ces recherches se sont focalisées sur les métropoles négligeant ainsi les autres espaces. L'objectif de cet article est de mieux comprendre le marché immobilier grâce à une démarche de déconstruction, puis de réarticulation. La déconstruction a consisté à identifier différentes logiques de marché en fonction du type d'objet (principalement des immeubles résidentiels), des acteurs et institutions, des contextes territoriaux et des temporalités, sur la base de recherches menées en Suisse. Une métasynthèse a été ensuite élaborée. Elle s'appuie sur les « trois étages » développés dans les travaux de Fernand Braudel (1985) qui rend compte dans ses travaux des activités économiques tant au quotidien que sur la longue durée, tant à l'échelle locale que planétaire.

Mots clés : Marché(s) immobilier(s), Braudel, Institutions, Territoires, Suisse.

Adresse email : thierry.theurillat@ unine.ch doi : 10.3166/ges.16.233-254 (C) 2014 Lavoisier, Paris. Tous droits réservés. 


\begin{abstract}
Summary
The real estate markets: actors, institutions and territories. Revealing the parties, the processes and the institutions and, consequently, both the diversity and contingency of the real estate markets, the existing increasing literature emphasises the contemporary numerous links and interdependencies between real estate, land value, planning and town planning policy and even the financial system. This paper is an attempt to understand all the real estate markets, from the most peripheral ones, where the urban rent is the lowest, to the most dense city centres. To gain a better understanding of the real estate market, a process of firstly deconstruction and then reconstruction is used. The process of deconstruction involves identifying various market trends according to property type (principally residential buildings), players and institutions, territorial situations and temporalities based on research conducted in Switzerland. We then developed a meta-synthesis inspired by Fernand Braudel (1985) whose works put as much emphasis on day-to-day economic activity as on long-term activity, and on local as well as global issues.
\end{abstract}

Keywords: Real estate market(s), Braudel, Institutions, Territories, Switzerland.

(c) 2014 Lavoisier, Paris. Tous droits réservés.

\title{
Introduction
}

Cet article est le résultat d'une insatisfaction. Lors de plusieurs recherches sur le marché immobilier, nous avons été frappés par l'inadéquation entre nos observations et la littérature. Sur le terrain, la diversité des situations est très grande. Quoi de commun entre une région rurale, dans laquelle les constructions sont généralement moins coûteuses, et le cœur d'une métropole, là où l'immobilier représente un capital considérable? Sur le plan théorique pourtant, l'immobilier a fait l'objet de nombreux travaux depuis les années 90 afin de dépasser la tradition néo-marginaliste (Wheaton, 1999) ou marxienne (Harvey, 1978 et 1985). Les interdépendances entre l'immobilier, les marchés financiers, l'aménagement urbain ou encore les politiques urbaines sont aujourd'hui de plus en plus traitées (Fainstein, 2001 et 2008; Aveline-Dubach, 2008; Theurillat, 2011a et b; David et Halbert, 2013b). Néanmoins, les études ont été avant tout centrées sur les métropoles, laissant de côté les villes plus petites et les régions non urbaines. Cet article présente une tentative de compréhension du marché immobilier d'une manière intégrée pour tous les niveaux de la rente urbaine, en allant des espaces les plus périphériques aux plus centraux.

On procédera par une démarche de déconstruction, puis de ré-articulation. La déconstruction a consisté à identifier différentes logiques de marché en fonction du type d'objet (principalement des immeubles résidentiels), des acteurs et institutions, des contextes territoriaux et des temporalités sur la base de recherches menées en Suisse. Nous avons ensuite élaboré une métasynthèse inspirée par Fernand Braudel (1985) qui rend compte dans ses travaux des activités économiques tant au quotidien que sur la longue durée, tant à l'échelle locale que planétaire.

Braudel distingue trois «étages » de la vie économique. Premièrement, l'étage de l'autoproduction et de l'autoconsommation, qui a largement dominé au cours de l'histoire, caractérise les situations où les ménages orientent leurs activités en fonction de leurs besoins ou aspirations, de la valeur d'usage des biens qu'ils produisent. Chacun 
produit pour soi ou via des relations de réciprocité au sein de communautés locales. Dans le domaine immobilier, il s'agit principalement des activités d'autopromotion, voire d'auto-construction.

Le deuxième étage, le marché, se caractérise par l'échange et la monnaie, et par une distinction entre producteurs et consommateurs. Les premiers orientent leurs actions non plus vers leurs propres besoins mais vers des objets et services ayant une valeur sur le marché (valeur d'échange). La monnaie est l'institution centrale qui permet la division technique et sociale du travail (Aglietta et Orléan, 1982). Dans l'immobilier, cet étage caractérise l'intervention de l'acteur professionnel, en particulier du promoteur qui calcule la différence entre les coûts monétaires de production et les prix du marché pour réaliser une marge. Ici, le marché est avant tout local et régional.

Enfin, au troisième étage, celui du capitalisme, les acteurs ne poursuivent plus une logique d'usage ou d'échange, mais recherchent la rentabilité d'un investissement en capital, un profit. Qui dit capital dit acteurs centralisant pouvoirs et moyens. La logique est moins celle du marché que de l'organisation. Contrairement au deuxième étage, où les prix s'imposent aux producteurs et aux consommateurs, les acteurs ont ici un certain pouvoir individuellement ou collectivement - pour influencer les prix et le cadre institutionnel de leurs activités. Dans l'immobilier, cet étage doit selon nous, être scindé en deux. En effet, certains acteurs sont orientés vers la production d'un parc immobilier « réel ». Ce sont les groupes de développement-construction qui agissent à l'échelle nationale voire internationale et qui développent de plus en plus de grands projets pour les investisseurs institutionnels. La stratégie financière de ces derniers est alors de tirer profit non pas du rendement des immeubles, mais des plus-values obtenues aussi bien par la croissance - à plus long terme - de la rente urbaine que par l'évolution - à court terme - des cours boursiers. Ce jeu d'opérateurs sur les marchés financiers constitue le « capitalisme immobilier financiarisé ».

Le chapitre suivant précise l'approche territoriale et institutionnaliste ainsi que les méthodes utilisées dans cet article. Puis, les trois étages du marché immobilier sont développés en mettant en évidence leurs dimensions sociales, institutionnelles et territoriales. Chaque étage est mis en relation avec la littérature. Afin d'illustrer le propos, des situations emblématiques rencontrées sur nos terrains sont analysées. Enfin, la cinquième partie montre comment ces logiques s'articulent de manière de plus en plus complexe en fonction du degré d'urbanisation.

\section{Une approche territoriale et institutionnaliste}

\subsection{Braudel et la littérature sur l'immobilier comme cadre conceptuel}

L'approche territoriale et institutionnaliste (Crevoisier, 2011) que nous proposons consiste à montrer les différentes formes de marchés de l'immobilier en lien avec le mode de financement en tenant compte des dispositifs techniques, des acteurs et des contextes institutionnels et territoriaux. Elle s'appuie sur des approches réalistes ou socioconstructivistes (Lawson, 1997; Sayer, 1992) des marchés immobiliers contemporains que nous réexaminons à la lumière des étages distingués par Braudel.

Afin de dépasser les approches abstraites et universalisantes, qui engendrent des travaux économétriques sur la constitution de portefeuilles d'investissement optimaux du côté néo- 
classique et qui conduisent à écraser le rôle des acteurs du côté marxiste ${ }^{1}$, différents auteurs se sont efforcés, à partir des années 90, d'ouvrir la boîte noire des marchés immobiliers. Certains ont tout d'abord mis en évidence les acteurs, institutions et processus relatifs au fonctionnement des marchés immobiliers, que ce soit dans une perspective institutionnaliste (Healey, 1991, 1992 et 1999; Ball, 1998; Keogh and D'Arcy, 1999; Guy and Henneberry, 2000) ou marxienne (Haila, 1991; Fainstein, 1994; Beauregard, 1994; Charney, 2001). Dès les années 2000, des auteurs ont traité des marchés immobiliers en inscrivant la production urbaine dans le système institutionnel néolibéral (Swyngedouw et al., 2002; Fainstein, 2008). L'immobilier, et plus précisément la transformation du paysage urbain à travers de grands projets, apparaît comme un objet d'analyse privilégié par une des littératures dominantes en études urbaines, dite de «l'actually existing neoliberalism» (Brenner et Theodore, 2002). Parallèlement, un nombre croissant d'auteurs abordent les interdépendances entre immobilier et finance en tenant compte de la diversité des contextes territoriaux dans une perspective institutionnaliste (Aveline-Dubach, 2008; Theurillat et al., 2010; Theurillat, 2011a et b; Theurillat et Crevoisier, 2012 et 2013) ou socioéconomique (Torrance, 2009; David et Halbert, 2013b).

Ces travaux soulignent la nécessité d' aborder la production de l'environnement construit à la lumière des liens entre le foncier, la finance ou encore les politiques publiques, et de ne plus placer l'immobilier «sous une cloche de verre pour mieux l'observer» (AvelineDubach, 2008 : 13). Ces liens sont mis en perspective ci-dessous à partir d'une interprétation et extension de la vision braudélienne des étages de la production économique. Il s'agit de clarifier la production de biens immobiliers principalement résidentiels dans des contextes territoriaux passant des zones rurales aux cœurs des métropoles ${ }^{2}$.

\subsection{Méthode et démarche}

Les trois formes idéales-typiques (étages) du marché de la construction (Tableau 1) sont développées à partir de la littérature et de trois recherches menées en Suisse.

La première a traité des relations entre capital financier et marchés immobiliers à travers trois grandes réalisations urbaines récemment achetées par des institutions financières (fonds d'investissement et caisses de pension) et localisées à Neuchâtel (ville moyenne), Berne (capitale politique) et Zurich (capitale économique). (Theurillat, 2011a; Theurillat et Crevoisier, 2012 et 2013).

\footnotetext{
${ }^{1}$ Dans les approches néoclassiques et marxistes, les marchés immobiliers dépendent de la croissance des autres secteurs économiques et ne sont pas considérés comme ayant des dynamiques endogènes. En d'autres termes, l'immobilier est une demande dérivée. Dans l'approche néoclassique, c'est du marché des biens que tous les autres dérivent. Une baisse sur ce marché affecte l'emploi, qui diminue la demande (bureaux et logements) et affecte in fine la rente. Dès lors, les développeurs réagissent à la demande mais avec un décalage dans les cycles conjoncturels en raison du temps nécessaire pour réaliser un projet. Dans l'approche marxiste, l'environnement construit, à savoir les infrastructures nécessaires à la production économique (usines, routes, etc.) et à la consommation (magasins, maison, etc.), constitue le deuxième des trois circuits d'accumulation et de circulation du capital. Harvey $(1978,1985$ et 2003) voit l'urbanisation du capital comme un passage du circuit primaire de la production vers celui de la propriété afin de surmonter la suraccumulation inhérente à la dynamique du capitalisme, c'est-à-dire la recherche de profits dans un environnement compétitif entre firmes capitalistes. Quant au troisième circuit, il est composé du secteur technologique (science et innovation) et du secteur social qui sont au service de la reproduction du capital. Le système financier joue un rôle majeur en faisant circuler le (surplus de) capital.

${ }^{2}$ Cet article n'aborde ainsi pas le marché secondaire (objets résidentiels déjà existants), ni les opérations de réhabilitation, ni l'immobilier commercial (bureaux, etc.).
} 
La deuxième a porté sur l'évolution démographique et l'attractivité résidentielle des villes suisses. Elle a étudié les acteurs (choix résidentiel des ménages, stratégie des acteurs privés et publics) impliqués dans des opérations de régénération urbaine à Neuchâtel et Zurich (Rérat 2010, 2012a, 2012b; Rérat et al., 2010 ; Rérat et Lees, 2011).

La troisième a abordé la mobilité résidentielle transfrontalière et le fonctionnement du marché immobilier dans l'Arc jurassien franco-suisse (Rérat et al., 2011). Du côté suisse, une attention particulière a été accordée à des villes industrielles petites (Le Locle) et moyennes (La Chaux-de-Fonds) et à des régions rurales (la Vallée de Joux).

Dans les marchés immobiliers étudiés, près de 90 entretiens ont été menés avec des acteurs clés et des observateurs privilégiés (gérances, entreprises de construction, banques, sociétés immobilières, investisseurs, pouvoirs publics, etc.).

Dans un deuxième temps, la comparaison des études de cas entre elles a montré que les différences ne relevaient pas uniquement de la spécificité des terrains mais de caractéristiques structurelles. Ceci suggérait l'existence de systèmes aux logiques de fonctionnement propres. À partir de ce constat, nous nous sommes inspirés des travaux de Braudel pour proposer un cadre conceptuel rendant compte de ces systèmes (les marchés immobiliers) tout en les intégrant dans un schéma global (le marché immobilier). C'est donc une démarche de métasynthèse (Finfgeld, 2003; Thorne et al., 2004; Walsh et Downe, 2005 ; Zimmer, 2006) qui, par un effort de généralisation obtenu par l'intégration progressive des études de cas, a permis l'élaboration d'un cadre interprétatif.

L'ambition du cadre interprétatif proposé est de s'extraire des particularités pour dégager un modèle global de compréhension et d'interprétation des mécanismes de la production de logements dans différents territoires. Il s'agit donc davantage d'une proposition conceptuelle issue d'une démarche idéal-typique qu'une restitution détaillée de différentes études de cas. Il n'en demeure pas moins que ce cadre interprétatif est influencé par le contexte suisse et certaines adaptations seraient nécessaires afin de l'appliquer à d'autres contextes nationaux.

Le marché immobilier helvétique présente certaines particularités. Tout d'abord, la Suisse se distingue par la proportion de propriétaires la plus faible d'Europe occidentale (Gerheuser 2004). Elle se monte à 34,6\% en 2000 alors qu'elle est supérieure à $50 \%$ dans les autres pays (à l'exception de l'Allemagne) et atteint parfois $80 \%$. Cette situation peut paraître paradoxale étant donné que la grande majorité des ménages affirme préférer la propriété à la location (Cuennet et al., 2002). Elle s'explique notamment par la cherté du foncier et de l'immobilier, le retard en matière de copropriété (interdite entre 1912 et 1965), l'apport personnel nécessaire à l'achat d'un bien (20\%), la bonne qualité du parc locatif et par la stabilité financière et monétaire (qui signifie que l'accession à la propriété n'est pas un moyen de se protéger contre l'inflation).

La majorité du parc immobilier locatif est aux mains de privés $(57,4 \%$ ) (Cuennet et al., 2002). Le reste appartient à des investisseurs (22,2\%, dont 8,4\% des caisses de retraite, 5,7\% des sociétés immobilières, $5,5 \%$ des assurances et 2,6\% des fonds de placement en immobilier), des coopératives $(7,9 \%)$ et d'autres institutions à but non lucratif $(5,9 \%$; dont 3,4 \% des collectivités publiques qui n'investissent quasiment pas dans l'immobilier; voir Cuennet et al., 2002). La part des organismes à but non lucratif, soit les deux dernières catégories de la liste, se monte donc à 13,8\%. À ce chiffre s'ajoute un parc social « de fait », soit des logements sur le marché libre à bas loyer en fonction du niveau de confort ou de la localisation. 


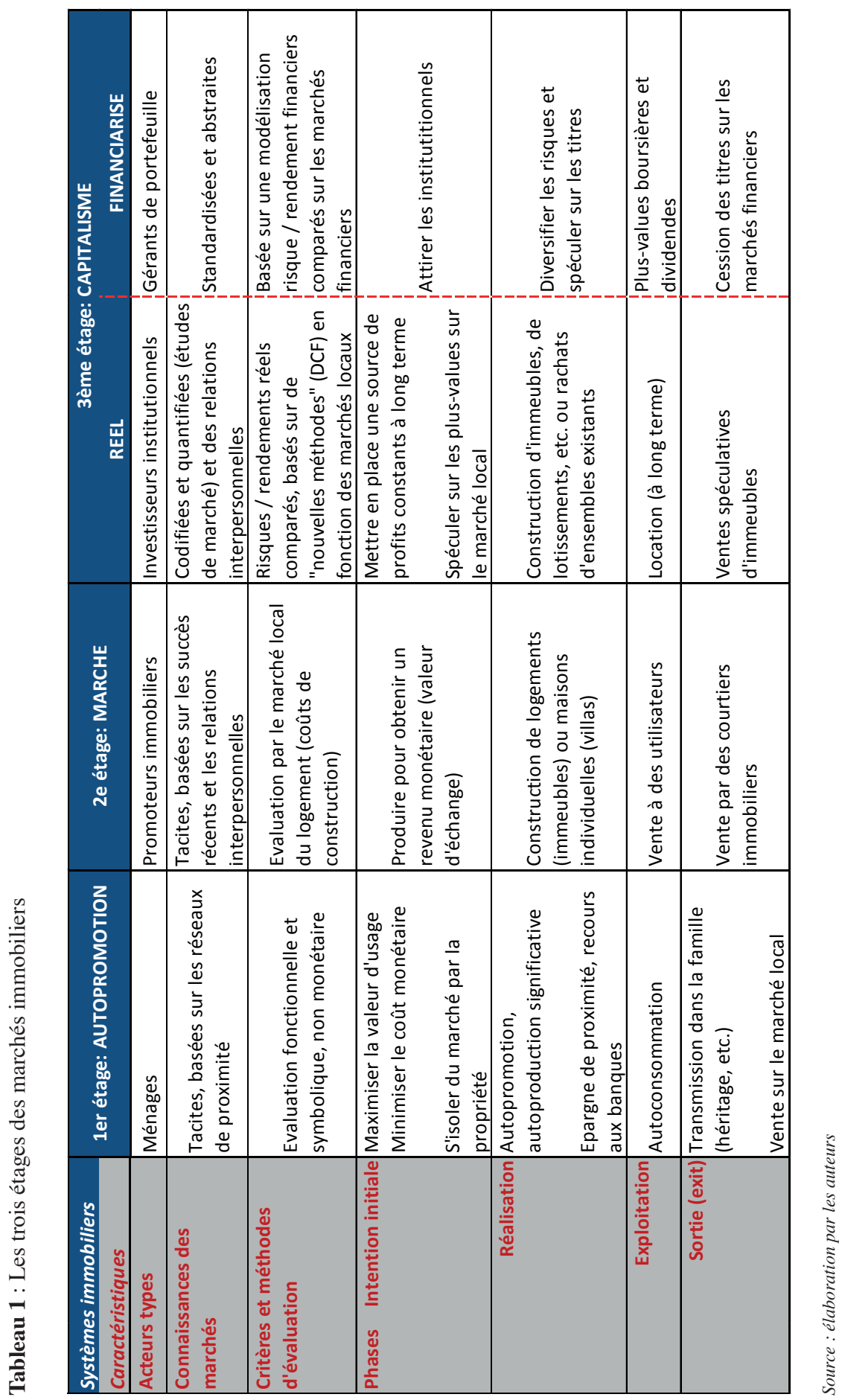




\section{2. Étage 1 : marché immobilier de l'autopromotion et de l'autoconsommation}

Dans ce premier étage, nous avons mobilisé la littérature abordant l'autopromotion et l'autoconsommation (soit le cas de figure où les occupants participent, de différentes manières, à la réalisation de leur logement). Cette forme de production occupe une place importante, voire dominante, dans de nombreux pays. Il ne s'agit pas uniquement de constructions informelles dans des pays du Sud (Aggier 1999; Bolay 2006) ou de slums dans certaines villes du Nord (Ascensão 2013). Bien qu'elle soit peu étudiée (voir toutefois Duncan et Rowe 1993; Dubois 2002; Halleux 2009), elle est également largement diffusée dans les espaces ruraux et périurbains des pays industrialisés à l'instar des communes de la Vallée de Joux.

\subsection{Caractéristiques de l'autopromotion dans l'immobilier}

\subsubsection{Acteurs, institutions et financement}

Dans cet étage, les promoteurs sont également les consommateurs. Duncan et Rowe (1993) distinguent l'autopromotion (self-provided housing) de l'auto-construction (self-build housing) même s'il existe des zones grises entre ces catégories. Dans l'autoconstruction, les personnes participent elles-mêmes à la construction de leur logement. L'autopromotion renvoie plus globalement aux cas où les ménages agissent comme promoteurs, investisseurs et usagers. Les démarches qu'ils entreprennent regroupent des aspects tels que la recherche d'un terrain, la constitution du capital ou les contacts avec la collectivité locale et les artisans.

Même dans des pays à revenus élevés, il est courant que des personnes investissent un temps important dans la construction ou la rénovation du logement qu'elles occuperont. Elles prennent ainsi en charge une partie de la conception et des travaux souvent avec l'aide de la famille et d'amis.

L'avantage de ce système est de minimiser les sorties monétaires (qui concernent avant tout les matériaux et le travail des artisans ou d'entreprises se rapprochant de l'étage 2) et l'endettement, tout en obtenant un logement pour son usage propre. L'autoproduction se caractérise par une faible division du travail et des compétences basses. Elle est guidée par la valeur d'usage.

Dans ce système, le financement repose sur différents piliers et varie selon les pays. Le premier est l'épargne du ménage et celle qu'il mobilise auprès de la famille (Poggio, 2012). L'apport de cette dernière peut également prendre la forme d'un héritage en nature (terrain ou immeuble) impliquant peu de transactions monétaires. Ensuite, mais ceci est déterminant, le financement monétaire est d'autant moins important que le ménage investit de son temps. Enfin, les prêts bancaires peuvent compléter le montage du projet.

\subsubsection{Spatialités et temporalités}

Cette logique de production a souvent pour objectif de garantir un habitat satisfaisant, de «sortir du marchand » en ayant un «chez-soi », un gage de sécurité indépendant de la conjoncture économique. La localisation dans des lieux périphériques, là où la rente urbaine est la plus faible, s'explique par les faibles capacités monétaires des acteurs impliqués, ainsi que souvent par l'absence de concurrence de la part des acteurs types des deux autres étages (promoteurs et investisseurs respectivement). 
Elle se retrouve prioritairement dans les espaces ruraux et périurbains et se matérialise par la construction de maisons individuelles ou de petits immeubles. Dans les villes, certains acteurs à but non lucratif - comme les coopératives - sont proches de l'autopromotion dans le sens où ils se placent hors marché et basent leur stratégie sur la valeur d'usage (voir le chapitre 5 pour plus de détails).

\subsection{Un exemple : les communes rurales de la Vallée de Joux}

L'autopromotion occupe une position dominante dans les zones rurales et périurbaines. Cette situation s'explique par les bas prix du foncier et de l'immobilier qui facilitent l'accès à la propriété, et par la faible présence des autres catégories d'investisseurs. Le cas de la Vallée de Joux permet de préciser cette forme de production de logements.

Le type d'objet construit est typiquement la maison individuelle. Il concerne en général des ménages vivant dans la région et souhaitant sortir du parc locatif pour accéder à la propriété. Leurs motivations renvoient à trois faisceaux de facteurs. Premièrement, l'achat d'une villa, avec le remboursement progressif de l'hypothèque, est perçu comme une épargne, par opposition au paiement d'un loyer, qui est considéré comme une dépense courante. Être propriétaire est également associé à une image de réussite sociale ainsi qu'à un mode d'appropriation de son logement («être chez soi »). Finalement, la villa constitue une forme d'habitat valorisée en particulier par les ménages avec enfants (jardin, etc.) ${ }^{3}$.

La récolte de l'information, l'aire de prospection, le montage du projet et les professionnels de l'immobilier mandatés s'inscrivent dans une échelle locale. Il en est de même pour le financement qui, en plus de l'apport personnel (les banques demandent généralement $20 \%$ du prix du bien en Suisse), implique un prêt hypothécaire. Les banques à fort ancrage régional détiennent l'essentiel du marché.

\section{3. Étage 2 : marché immobilier urbain}

Le deuxième étage est le marché immobilier stricto sensu et s'organise autour de la valeur d'échange. Cette partie s'appuie sur un deuxième corpus qui, d'une part, comprend une perspective néo-marxiste déconstruisant le marché de l'immobilier et des infrastructures - soit le deuxième circuit du capital décrit par Harvey (1978, 1985, 2003) - et analysant son fonctionnement (Haila, 1991; Fainstein, 1994; Beauregard, 1994; Charney, 2001). D'autre part, la littérature utilisée se réfère à des auteurs socio-institutionnalistes (Healey, 1991, 1992, 1998 et 1999) ou institutionnalistes (Ball, 1998; Keogh and D’Arcy, 1999; Guy et Henneberry, 2000) qui, en étudiant les institutions, processus et jeux des acteurs, analysent notamment le rôle crucial des promoteurs-constructeurs. Cet étage, caractéristique des régions urbaines, est illustré par deux villes moyennes : La Chaux-de-Fonds et Neuchâtel.

\footnotetext{
${ }^{3}$ On retrouve ainsi les principales motivations mises en évidence par d'autres études (voir par exemple Haumont 2001 et Bourdieu et al., 1990).
} 


\subsection{Caractéristiques du marché des biens immobiliers}

\subsubsection{Acteurs, institutions et financement}

Le marché est une institution qui a pour principale caractéristique de distinguer socialement les producteurs spécialisés des consommateurs, et de les réunir par différents mécanismes de négociation et d'échange. Dans le secteur immobilier, il correspond à l'émergence de promoteurs et courtiers professionnels.

Le marché se base sur le calcul, par le promoteur, de la différence entre les coûts monétaires de production et les prix du marché dans le but de dégager une marge. Cette marge lui permet de rémunérer son travail, voire de dégager un profit supplémentaire. Ce dernier peut résulter d'un mauvais fonctionnement du marché (hétérogénéité des biens, absence de transparence notamment). Le marché se caractérise aussi par la concurrence et le fait que les prix s'imposent aux producteurs comme aux consommateurs. Les producteurs ne parviennent à les influencer qu'à la marge. Il arrive donc également que des producteurs inefficaces soient évincés du marché.

Le marché immobilier urbain repose prioritairement sur la construction de petits immeubles (voire de maisons) dont les appartements sont destinés à des particuliers. Ces derniers jouent à la fois le rôle de financeurs, de propriétaires et d'usagers. Ce marché est basé sur la valeur d'échange et organisé à une échelle locale. Dès lors, les acteurs clés sont les sociétés immobilières et les entreprises de construction qui disposent de connaissances tacites d'un marché particulier. Leurs profits dépendent de la demande, du revenu et de l'épargne des particuliers, ainsi que des conditions relatives aux prêts hypothécaires qui relèvent du cadre institutionnel du marché de la propriété individuelle.

Dans le cadre de la dynamique intrinsèque des marchés immobiliers, nombre d'auteurs insistent sur le rôle des agents, en l'occurrence des promoteurs et entrepreneurs immobiliers (Haila, 1991; Healey, 1992). Alors que les objets qu'ils réalisent s'inscrivent dans un marché, ils développent des stratégies afin de répondre à la demande existante, mais aussi de se positionner face à la concurrence par des produits novateurs. À cet égard, Charney (2001) montre les trois dimensions sur lesquelles les entrepreneurs jouent dans la constitution de l'offre. Ils peuvent se spécialiser en combinant la localisation, les secteurs (résidentiels, commerciaux, industriels ou de bureaux) et les objets (bâtiments neufs ou existants).

Ensuite, le rôle de la connaissance spécifique des institutions et des marchés immobiliers locaux est à souligner. L'action et la « rationalité » des entrepreneurs s'inscrivent dans un cadre institutionnel donné (réglementations en matière de construction et d'aménagement, politiques en matière de promotion économique, de développement durable, etc.) et dans un ensemble de relations formelles (contrats) et informelles (connaissances interpersonnelles). Sur cette base s'élaborent divers arrangements autour de la production immobilière (Guy et Henneberry, 2000; Fainstein, 2001; Healey, 1999; Healey et al., 2002).

\subsubsection{Spatialités et temporalités}

Les marchés immobiliers du deuxième étage s'organisent sur une base locale ou régionale pour plusieurs raisons. Premièrement, la demande est en principe structurée par le fait que les ménages cherchent une possibilité de logement là où ils disposent d'un emploi, c'est-à-dire dans un périmètre représentant un temps de déplacement domicile-travail 
acceptable. La demande se caractérise également par un capital économique variant fortement selon les régions. Ensuite, les marchés immobiliers sont souvent opaques en raison de l'hétérogénéité des biens, de l'importance déterminante des régulations et spécificités locales et du rôle des politiques locales. Tout cela conditionne l'offre ainsi que le niveau des prix du terrain. Il en va de même pour les différents services et infrastructures auxquels les ménages ont accès. Ces marchés immobiliers se caractérisent par une logique au coup par coup; la grande force des promoteurs locaux est précisément de savoir identifier et exploiter au mieux les occasions qui se présentent.

Le marché immobilier constitue un des principaux circuits économiques locaux et régionaux. Il s'agit en effet d'une dépense importante dans le budget d'un ménage et dont bénéficient les propriétaires fonciers et immobiliers, les promoteurs et entrepreneurs généralement à fort ancrage local. Ces acteurs distribuent ensuite des salaires à des travailleurs locaux, si bien que l'immobilier peut engendrer un circuit économique régional important et contribuer à la compétitivité régionale (D’Arcy et Keogh, 1999).

\subsection{Un exemple : les villes moyennes de Neuchâtel et de La Chaux-de-Fonds}

Lorsque la rente foncière devient trop élevée, les autopromoteurs sont remplacés par des sociétés immobilières et des entreprises de construction, qui ne se contentent pas de la réalisation stricto sensu mais endossent un rôle de promoteur. Elles occupent ainsi une part de marché importante dans des villes moyennes comme Neuchâtel et La Chaux-de-Fonds.

Leur objet de prédilection sont des immeubles regroupant généralement au plus une vingtaine de logements et issus par exemple d'opérations de densification. Il peut parfois s'agir de lotissements de villas aux marges du tissu urbain. De telles opérations sont trop importantes pour des particuliers mais trop réduites pour les investisseurs de l'étage 3 .

Ces sociétés poursuivent deux objectifs : générer du travail pour leur propre entreprise et dégager rapidement une marge en vendant les objets construits. Plusieurs raisons expliquent leur préférence pour la vente par rapport à la mise en location : des perspectives de rendement supérieures, le travail de gestion coûteux en temps et en argent et le risque de logements vacants. Les investisseurs finaux sont des particuliers si bien que ce type de maître d'ouvrage se trouve à l'origine de la plupart des logements en copropriété. Ils répondent ainsi à une demande croissante soutenue par des taux hypothécaires particulièrement bas ces dernières années en Suisse et qui facilitent l'accession à la propriété. Certaines sociétés ont toutefois une taille suffisante pour initier des projets de grande envergure (une cinquantaine de logements) qu'elles vendront ensuite clé en main aux investisseurs de l'étage 3 (voir plus bas).

Les entreprises de construction et sociétés immobilières actives à Neuchâtel et La Chauxde-Fonds y sont localisées. Par leur ancrage territorial, elles connaissent le marché et ses tendances, sont en contact fréquent avec leurs partenaires et concurrents et connaissent le degré de succès des projets réalisés. Elles agissent plutôt au coup par coup, lorsqu'elles repèrent une occasion grâce à leur connaissance du terrain et leurs réseaux locaux. Les expressions «feeling du marché » ou «sentir la demande» sont apparues fréquemment lors des entretiens. Cette approche se substitue aux études de marché pour déterminer la demande et ceci d'autant plus que la taille des projets est généralement réduite. 


\section{4. Étage 3 : marché immobilier métropolitain}

Le troisième étage renvoie à des investisseurs par métier plaçant leurs capitaux dans l'immobilier avec l'objectif de faire du profit. La propriété revient au détenteur des capitaux; elle est dissociée de l'usage des objets qui sont en principe mis en location. La production d'un bien immobilier n'a pas pour objectif de permettre au capitaliste d'obtenir un revenu pour consommer, mais de reproduire le capital investi. Bien entendu, il s'agit là d'une distinction conceptuelle. Dans la réalité, il peut arriver que les rôles de capitaliste, de promoteur, de constructeur, voire d'usager, se superposent.

La littérature relative à cet étage porte essentiellement sur les investisseurs institutionnels s'intéressant à l'immobilier et aux infrastructures des métropoles. Deux types de capital financier sont à distinguer : le capitalisme immobilier réel qui se matérialise par des investissements concrets et le capitalisme immobilier financiarisé qui prend la forme d'investissement dans des titres cotés ou non sur les marchés financiers. Dans ce dernier cas, les investissements reposent sur une logique de portefeuille et ne se traduisent pas forcément par des réalisations concrètes. Ce double type de marché est illustré par Zurich, métropole financière d'importance mondiale.

\subsection{Caractéristiques du capitalisme immobilier « réel»}

\subsubsection{Acteurs, institutions et financement}

L'immobilier et plus largement la ville ont depuis longtemps attiré le capital d'institutions financières (Harvey, 1985 : Fainstein, 2001) telles que les banques et les assurances. Ces dernières sont intéressées par les revenus provenant de la valeur locative (à long terme) et/ou par la valeur spéculative (revente avec plus-value).

Depuis la crise financière de 2001, les institutions financières montrent un intérêt renouvelé pour les placements dans l'environnement construit et ceci principalement dans les métropoles. Cet intérêt résulte d'une part de réformes institutionnelles concernant les marchés financiers et le développement de l'épargne collective gérée de manière centralisée par des investisseurs institutionnels ${ }^{4}$. D'autre part, il découle de la mise à disposition d'objets suite à la privatisation et à l'externalisation de la propriété immobilière d'entreprises et d'institutions publiques dans de nombreux pays (Aveline-Dubach, 2008; Theurillat, 2011b). Dès lors, les revenus immobiliers de la location résidentielle sont de plus en plus complétés par ceux de l'immobilier d'entreprise et de grands objets sous la forme de complexes multifonctionnels (Theurillat, 2011a), de projets de revitalisation urbaine (Hagerman et al., 2007) ou d'infrastructures (Torrance, 2009).

La matérialisation du capital financier dans la ville se réalise par des acteurs spécifiques : de grands groupes de développement-construction d'envergure nationale, voire internationale, pouvant être cotés en Bourse et, selon les pays, adossés à des banques, font l'interface avec les investisseurs institutionnels. Dans le cas de projets urbains d'envergure, ces groupes jouent un rôle central en tant qu'intermédiaires entre les investisseurs

${ }^{4}$ Le capital financier provient de l'épargne, notamment de retraite, des pays riches et de plus en plus de pays émergents (fonds de pension par exemple) ou du déséquilibre des balances commerciales (fonds souverains). Cela a engendré la création et la multiplication de fonds et produits financiers dans différents secteurs de l'économie (fonds en private equity d'entreprises, hedge funds, fonds de produits dérivés, etc.) et en particulier dans l'immobilier. 
institutionnels et les territoires. D'une part, ils disposent des compétences aussi bien techniques que dans le montage juridique et financier et d'autre part, ils ont des connaissances spécifiques et souvent tacites des marchés immobiliers locaux (Wood, 2004; David et Halbert, 2013a; Theurillat, 2011b; Theurillat et Crevoisier, 2013). En étant directement impliqués dans les activités de construction, ils ont également des relations interpersonnelles avec les différentes parties prenantes (propriétaires, municipalités, partis politiques, exploitants commerciaux, futurs utilisateurs, etc.). Les acteurs institutionnels font également appel à certains analystes et experts immobiliers qui évaluent les investissements et produisent des informations quantitatives et à visée comparative entre objets immobiliers et villes ou localisations potentielles.

\subsubsection{Spatialités et temporalités}

L'étage capitaliste, dans l'immobilier comme ailleurs, se caractérise avant tout par l'importance des montants monétaires, la taille des opérations concernées et la localisation systématique dans les lieux où la rente urbaine est la plus élevée. L'investissement est une dépense effectuée à un moment donné afin de dégager des revenus au cours d'une certaine période future. On est donc bien dans un circuit totalement monétarisé, tant du côté de la production que de la consommation ${ }^{5}$.

L'espace d'investissement du capitalisme réel concerne prioritairement les métropoles et les grands objets immobiliers (Theurillat et al., 2010; Torrance, 2008; Halbert, 2004). Le capital provient principalement des fonds immobiliers, sociétés immobilières (Real Estate Investment Trusts) et fonds de pension domestiques ou étrangers. Certains auteurs (David et Halbert, 2010; Sassen, 2010) soulignent les liens entre mondialisation des firmes, financiarisation et métropolisation. La mondialisation des firmes a accru la demande pour des immeubles de bureaux et des infrastructures répondant aux standards des grandes entreprises, dopant ainsi les marchés métropolitains alimentés par le capital financier international. Il s'ensuit que la rente du capitalisme immobilier réel concerne des secteurs bien particuliers des grandes villes (Lizieri, 2009). Les investisseurs institutionnels misent ainsi sur une rente urbaine, à plus ou moins long terme dans le cas de revenus locatifs et à plus ou moins court terme dans le cas de plus-values escomptées avec la (re)vente d'objets immobiliers.

Le changement d'échelle des marchés immobiliers et la connexion avec le capital financier national et étranger se manifestent également au niveau des intermédiaires spécialisés (Magalhaes, 2002). Dans ce marché, on trouve de grands groupes de la promotion-construction et des sociétés de conseils en immobilier actives à une échelle internationale (Cushman and Wakefield, Jones Lang Lasalle, etc.). Afin de qualifier ces réseaux d'acteurs faisant circuler le capital à plus ou moins longue distance jusqu'à son ancrage effectif dans l'environnement urbain, Halbert et Rouanet (2013) proposent la notion de « réseaux territoriaux transcalaires ». Les institutions financières s'appuient ainsi sur des professions spécialisées dont la fonction consiste à connecter le capital au territoire urbain. Les informations produites par différentes entreprises de consultance et de notation spécialisées sont formatées afin de permettre aux investisseurs, qui considèrent le

\footnotetext{
${ }^{5}$ Au premier étage, la monétarisation est très partielle. Au deuxième, les acheteurs-usagers peuvent rembourser leurs dettes et ainsi se détacher presque complètement des circuits monétarisés.
} 
territoire « à partir du ciel », de sélectionner les projets (Theurillat et Crevoisier, 2013). En effet, contrairement aux usagers, qui sont ancrés dans une région, aux promoteurs, qui dépendent de leurs connaissances du marché local, les investisseurs procèdent à une évaluation comparative des espaces. Cette intermédiation spécialisée est à la base des modèles d'investissement dits de discounted cash flows (DCF) utilisés par les investisseurs institutionnels et dans lesquels les caractéristiques des immeubles et marchés locaux sont standardisées et quantifiées pour permettre la comparaison, la hiérarchisation et le suivi des investissements.

\subsection{Caractéristiques du capitalisme immobilier «financiarisé »}

\subsubsection{Acteurs, institutions et financement}

L'intérêt renouvelé des investisseurs institutionnels pour l'immobilier se manifeste également sur les marchés financiers. En effet, la financiarisation à grande échelle qui a touché les activités industrielles, financières et les matières premières depuis vingt-cinq ans a aussi atteint l'immobilier. Avec la crise boursière de 2001, l'immobilier est même devenu extrêmement prisé contribuant, dans la logique de portefeuille (Markowitz, 1959) à diversifier et à diminuer le risque. Dès lors, une logique financière a présidé à l'évolution de ce secteur. Des promoteurs, alliés à des sociétés financières, ont proposé des projets de grande ampleur (Espagne, Irlande, etc.), ou la financiarisation d'immeubles existants (Hollande et États-Unis avec les subprimes).

Dans ce segment, la logique purement financière a souvent dominé la logique réelle de l'immobilier. Ainsi, des villes entières ont été construites en Espagne sans qu'elles soient ni vendues ni louées (Vorms, 2009). Comme les investisseurs sur les marchés financiers misaient massivement sur la croissance du secteur (également par anticipations mimétiques), les cours poursuivaient leur ascension, indépendamment des réalités locales. Il s'agit donc de phénomènes financiers aux conventions spécifiques (Orléan, 2011), c'est-àdire reposant sur des perceptions de l'évolution d'un secteur économique partagées au sein de la communauté financière. La convention des dot.coms dans les années 90 et celle de l'immobilier dans les années 2000 en sont deux exemples emblématiques. Les profits des institutionnels proviennent de plus-values financières réalisées à court terme (vente/achats).

La financiarisation du marché immobilier a été rendue possible grâce au développement de la liquidité/mobilité du capital (Crevoisier et Corpataux, 2005 ; Corpataux et al., 2009), découlant de la titrisation et de l'apparition de véhicules d'investissement immobiliers - fonds et sociétés cotés ou non ${ }^{6}$ sur les marchés financiers - dans lesquels des institutionnels peuvent acheter des parts. L'évolution des marchés immobiliers financiarisés dépend ainsi d'autres secteurs et espaces en fonction des opinions dominantes des opérateurs financiers.

Comment ces investissements, massifs, tout particulièrement depuis l'intérêt renouvelé des financiers pour le construit urbain, soit entre 2000 et 2008 , se sont-ils traduits dans l'immobilier réel? En d'autres termes, quelle est l'articulation entre capitalisme immobilier réel et financiarisé? Cette question n'a pour l'instant pas été traitée de manière

\footnotetext{
${ }^{6}$ Il existe en effet de nombreux marchés financiers non cotés rassemblant des institutions financières. Les prix sont négociés de gré à gré et la liquidité/mobilité (exit) est moins facile.
} 
approfondie. Nous pouvons cependant émettre l'hypothèse, qu'à l'instar des entreprises de production de biens et services, il existe un certain nombre de «fuites » liées à la réinjection du capital sur les marchés financiers (hausse purement spéculative des cours indépendante des réalisations concrètes). Le capital financier ne se matérialise pas automatiquement en investissements concrets mais est utilisé pour diverses opérations faisant monter les cours, pour les actionnaires (opération de relution), pour les gérants de portefeuilles (stock-options) ou encore pour les entreprises afin de faciliter le rachat d'entreprises concurrentes (fusions/acquisitions).

\subsubsection{Spatialités et temporalités}

Un «espace financiarisé » (Corpataux et al., 2009; Theurillat, 2011a) rassemble les principales places financières du monde qui forment la «global city » (Sassen, 1991). C'est entre elles que le capital circule à grande vitesse. En effet, le capital des ménages levé par exemple à des fins de retraite, est ensuite réinvesti dans les circuits financiers. Une partie de ces capitaux génèrent des projets localisés à plus ou moins longue distance des épargnants voire dans d'autres pays.

Dans le circuit financiarisé, les gérants de fortune se livrent à une comparaison instantanée des produits et espaces financiers. L'évolution de l'immobilier dépend donc étroitement des informations sur l'évolution (anticipée et mimétique) des cours d'autres actifs financiers.

\subsection{Un exemple : la métropole financière de Zurich}

Traditionnellement les caisses de pension et assurances helvétiques sont des investisseurs immobiliers. Elles possèdent des immeubles, essentiellement résidentiels, localisés dans leur région d'implantation pour les caisses de pension moyennes et petites, ou plus disséminés pour les plus grandes (Theurillat, 2010). Depuis les années 2000, les fonds immobiliers (appartenant notamment aux principales banques du pays) et de grandes sociétés cotés en bourse ont connu un succès important, l'immobilier financiarisé étant devenu un produit recherché par les caisses de pension en raison de sa facilité d'acquisition et de gestion.

Zurich est un exemple emblématique du captage du capital financier dans le construit urbain. Les principaux fonds immobiliers helvétiques, tous localisés à Zurich, ont développé de nouveaux produits financiers qui ont permis la conception et la réalisation de grands projets multifonctionnels accueillant habitat, bureaux et surfaces commerciales. C'est le cas par exemple de Sihlcity (centre commercial), de Greencity ${ }^{7}$ et de plusieurs projets dans le périmètre de Zurich West qui mêlent habitat et activités économiques (Puls5) ou bureaux et commerces (Swiss Prime Tower). Ces opérations d'envergure, jusqu'à plusieurs centaines de millions de francs suisses, ont permis la régénération de vastes friches industrielles.

En tant que place financière mondiale, Zurich gère des flux financiers importants. Les fonds immobiliers sont en mesure de drainer du capital à l'échelle nationale (caisses de

\footnotetext{
${ }^{7}$ Ce quartier, d'un coût de 800 millions CHF, est basé sur les énergies renouvelables et construit sur une ancienne friche. Il comporte des surfaces commerciales, des bureaux, des appartements en location et en coopérative.
} 
pension et assurances) et globale (investisseurs institutionnels et particuliers étrangers) ${ }^{8}$ et de l'investir en bonne partie dans la métropole zurichoise, devenue le lieu emblématique de l'investissement financiarisé dans le construit urbain en Suisse.

Tout un système industriel, financier, juridique et politique s'est mis en place dans les années 2000. Il regroupe les principales institutions financières immobilières de la place (dont certaines endossent les rôles de promoteur et d'investisseur et conservent parfois les immeubles dans leur portfolio) et les grandes entreprises de promotion-construction nationales (qui développent des projets destinés à être vendus aux institutionnels ou qui construisent les projets développés par ces derniers). On trouve également dans le marché zurichois de grandes entreprises louant d'importances surfaces de bureaux ou commerciales (sociétés d'assurance, banques, chaînes de supermarché, etc.) et les principales sociétés de conseils en immobilier.

Ce système comprend également la Municipalité de Zurich qui a adopté dans les années 1990 une nouvelle stratégie en matière d'urbanisme basée sur la collaboration avec les différents acteurs impliqués dans les projets (propriétaires fonciers, investisseurs, etc.) (Rérat et al., 2010). Cette stratégie a été appliquée en premier lieu dans le cadre de la régénération de friches industrielles et s'est inscrite dans une volonté d'augmenter la population de la ville après quelques décennies de recul démographique (Rérat 2012).

Zurich est un lieu incontournable en Suisse de l'ancrage du capital financier provenant d'autres régions ou de l'étranger. La rente urbaine est donc largement fonction du secteur de la finance qui est à même d'agir sur cette rente. Les anticipations mimétiques des marchés financiers relatives tant à la finance qu'à l'immobilier trouvent ici un prolongement dans le construit urbain réel et l'évolution de ce dernier influence, partiellement, la bonne santé du secteur financier. Dans le cas de Zurich, le secteur financier et l'immobilier, qu'il soit titrisé ou non, sont donc étroitement liés. On a donc une logique circulaire, autoréférentielle, centrée sur la Global city et drainant l'épargne des périphéries.

\section{La dynamique des marchés immobiliers selon les contextes institutionnels et territoriaux}

Les trois étages présentés sont des outils conceptuels et analytiques, des idéaux-types de systèmes de production immobilière (Tableau 1). L'objectif de cette dernière partie est de réunir ces trois logiques et d'esquisser la manière dont elles s'articulent selon le degré d'urbanisation puis dans le cadre des dynamiques de gentrification.

\subsection{Superpositions des logiques et concurrence pour la rente}

Dans les trois formes idéales-typiques des systèmes immobiliers, le premier étage se réfère en priorité aux espaces ruraux, le deuxième aux espaces urbains en général et le troisième aux espaces métropolitains. Ce gradient qui va d'espaces à faible densité jusqu'aux centres des plus grandes villes rend compte du phénomène organisateur central des marchés immobiliers dans l'espace, à savoir la rente urbaine. La rente correspond à la

\footnotetext{
${ }^{8}$ Le cadre législatif fédéral limite toutefois l'apport de capital en provenance de l'étranger pour les immeubles résidentiels («Lex Koller») : le portfolio des fonds cotés en bourse et ouverts aux investisseurs étrangers ne doit ainsi pas comprendre plus de $20 \%$ de logements.
} 
capitalisation sur le prix du sol, puis de l'immobilier et des loyers, des avantages procurés par une localisation dans des lieux centraux (Camagni 1996). Précisons que la rente ne doit pas être confondue avec le profit. Le profit est la rémunération du capitaliste, une rémunération qui correspond à l'avance faite et aux risques encourrus. La rente, dès sa conceptualisation par Ricardo, est un revenu qui ne correspond ni à la rémunération d'un travail, ni du capital. C'est un phénomène qui résulte d'un positionnement privilégié, par exemple au centre d'une ville, lorsque des facteurs généraux changent, et que cette ville, pour de multiples raisons, connait une croissance. Dans ce cas les détenteurs des terrains centraux profiteront d'un avantage sans avoir livré de contrepartie. La lutte pour l'appropriation de cette rente par les acteurs économiques conduit à une pression d'autant plus forte que l'on se rapproche du centre. Ceci explique l'accroissement de la densité, ainsi que celle de la création de valeur monétaire au mètre carré. La Figure 1 reprend ces distinctions tout en soulignant leur superposition croissante avec le degré d'urbanité.

Les métropoles sont emblématiques de cette imbrication. On y trouve aussi bien le marché, basé sur la valeur d'échange, que le capitalisme immobilier, basé sur les profits et la rente urbaine réelle ou financiarisée. Le premier étage, basé sur la valeur d'usage et sur une logique de mise à l'écart du marché, est également présent avec des organismes à but non lucratif tels que les coopératives ${ }^{9}$. Elles partagent également avec l'autopromotion classique l'implication directe des futurs habitants dans la réalisation et la gestion d'un bien immobilier. Leur mise en place est encouragée afin de répondre à des objectifs d'ordre social (les loyers sont inférieurs au marché libre) mais également environnemental (critères de construction). En raison du phénomène de la rente urbaine, les habitants à faible revenu sont repoussés dans les périphéries. Une politique publique est donc indispensable si l'on souhaite conserver une diversité sociale dans les lieux centraux ${ }^{10}$.

En Suisse, les coopératives sont au cœur de la nouvelle politique du logement adoptée par la Confédération et sur laquelle se calquent les politiques des Cantons. Elles ont le soutien des autorités par la mise à disposition de terrains, le cautionnement de prêts hypothécaires, etc. Le montage financier des projets se base notamment sur les fonds propres constitués des parts sociales versées par chaque membre de la coopérative. Le statut d'occupation est hybride («locataire-propriétaire ») : un ménage ne possède pas son appartement en tant que tel mais une partie du projet dans son ensemble. En Suisse, les coopératives possèdent plus du vingtième du parc de logements. Leur implantation, malgré le soutien politique récent, correspond encore aux lieux d'émergence du mouvement coopératif au début du $\mathrm{XX}^{\mathrm{e}}$ siècle, tels que Zurich où plus de $20 \%$ des logements se trouvent entre leurs mains.

\footnotetext{
${ }^{9}$ Les principes des coopératives ont été appliqués à l'habitat en premier lieu en Allemagne et dans les pays voisins (Autriche, Danemark, Pays-Bas, Suède, Suisse) (Kemeny et al., 2005).

${ }^{10}$ On pourrait également associer à cet étage des fondations ou des agences détenues ou soutenues par les collectivités publiques ou ces dernières elles-mêmes. Cette association n'est toutefois que partielle dans le cas suisse. En effet, les villes détiennent des parcs de logements dont seule une partie est subventionnée, le reste étant géré selon des critères usuels de rentabilité (Cuennet et al., 2002). De même, les collectivités publiques peuvent encourager des maîtres d'ouvrage de tous types qui en échange « acceptent de se soumettre à certaines conditions concernant la rentabilité des immeubles et donc le niveau des loyers. [...] Il serait donc erroné [en Suisse] d'assimiler le logement subventionné à du logement public et le logement public à du logement social » (ibid., 15-16). De plus, selon sa part, le logement d'utilité publique peut influencer, mener ou dominer l'ensemble du marché locatif. En Suisse, il n'a qu'une influence limitée (Kemeny et al., 2005).
} 
Les villes, petites et moyennes, sont également susceptibles d'attirer des investisseurs institutionnels locaux ou nationaux (troisième étage) dans le cas de grands objets. En Suisse, des projets résidentiels d'envergure et des complexes multifonctionnels (stades combinés à des centres commerciaux) ont par exemple été construits dans ces dernières et acquis par des fonds immobiliers ou caisses de pension (Theurillat, 2011a).

Une superposition existe également dans les espaces ruraux entre les premier et deuxième étages. Elle est plus rare avec le troisième même si certaines stations alpines de réputation internationale parviennent à attirer le «grand » capital. L'apparition de mégaprojets touristiques dans les Alpes représente des opportunités de diversification pour les fonds suisses et étrangers, le marché immobilier helvétique étant réputé stable et sûr.

Figure 1 : Les territoires des marchés immobiliers et la concurrence pour la rente urbaine

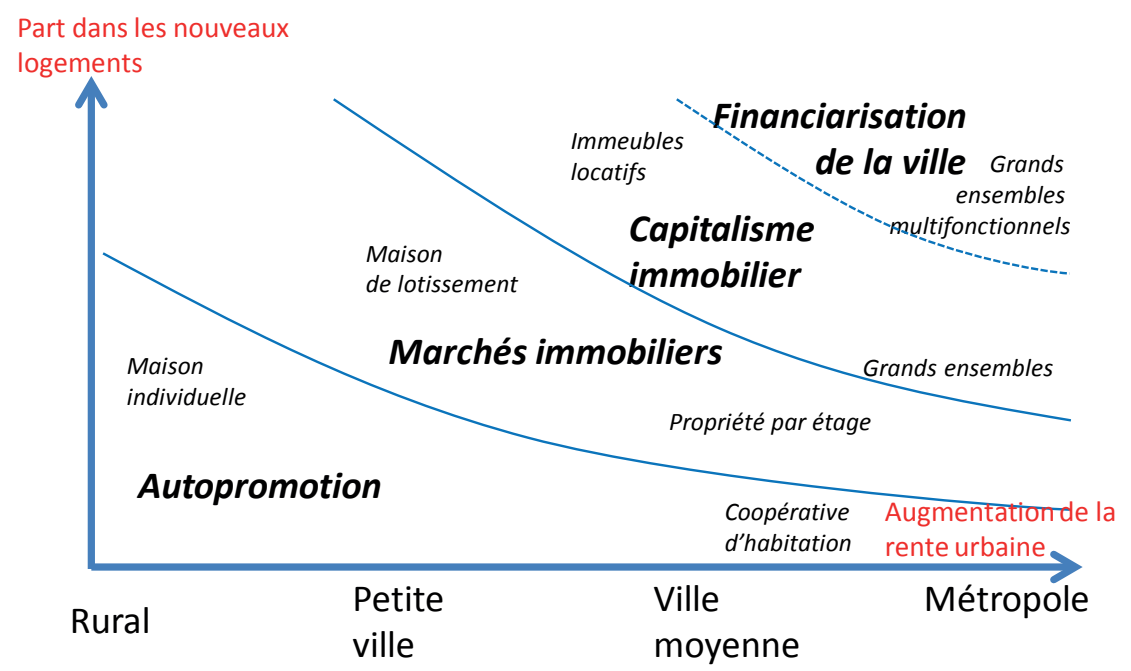

Source : élaboration par les auteurs

\subsection{La gentrification comme complémentaritélopposition entre les trois logiques}

La gentrification exemplifie l'articulation entre les trois étages. Ce phénomène peut être défini comme la transformation de quartiers urbains par/pour les classes moyennes supérieures. Documentée d'abord dans des métropoles telles que Londres, la gentrification s'est diffusée à d'autres contextes territoriaux et échelons de la hiérarchie urbaine, si bien qu'elle est devenue une tendance marquante du changement urbain. Les diverses formes de gentrification partagent quatre caractéristiques (Lees et al., 2008 ; Rérat et al., 2010) : le réinvestissement en capital dans certains secteurs urbains, l'arrivée de groupes au statut socio-économique supérieur, des transformations du paysage et de l'environnement construit, et des effets d'éviction (directs ou indirects).

Selon les formes et étapes de gentrification, le réinvestissement en capital renvoie à l'un ou l'autre des étages identifiés. La gentrification est souvent initiée par des ménages pionniers et notamment des artistes qui ont la faculté de dénicher des secteurs bon marché 
mais à grand potentiel (Ley, 2003). Ces acteurs ont par exemple créé de nouveaux modes d'habitat comme les lofts (Zukin, 1982). Dans cette phase, l'investissement est limité financièrement mais important en termes de temps de travail des utilisateurs (on parle alors de sweat equity).

La revalorisation du quartier et de son image se traduit à terme par une augmentation des prix de l'immobilier qui provoque le déplacement des premiers gentrifieurs et l'arrivée de ménages au capital économique supérieur. Dans cette phase, les acteurs clés sont les promoteurs dont Smith (1979) a interprété le rôle comme le repérage et l'exploitation du rent gap (soit la différence entre le rendement actuel et le rendement potentiel en cas de redéveloppement). Il considère d'ailleurs la gentrification avant tout comme un retour en ville du capital et la conséquence de processus de désinvestissement et réinvestissement.

La gentrification peut également prendre la forme de projets de grande ampleur lorsque des promoteurs et investisseurs transforment des quartiers entiers parfois avec le soutien des pouvoirs publics (Hackworth et Smith, 2001). De telles opérations nécessitent d'importants volumes de capital si bien qu'elles révèlent les liens entre acteurs globaux, financiarisation de l'immobilier et gentrification.

Dans les deuxièmes et troisièmes étages, les capitaux nécessaires proviennent de promoteurs et d'investisseurs et ce n'est que dans un deuxième temps que les ménages participent au réinvestissement des zones centrales par l'intermédiaire du paiement de leur loyer ou de l'acquisition d'un logement. Alors que la première forme implique une participation directe des gentrifieurs dans la rénovation de leur logement, les autres représentent une version « commodifiée » de la gentrification (Butler, 1997).

\section{Conclusion}

Depuis les années 90, des chercheurs ont déconstruit le marché immobilier dans le but d'appréhender ses dynamiques endogènes. Ces travaux ont mis en évidence les acteurs, les processus et les institutions et par conséquent la diversité ainsi que les contingences des marchés immobiliers. De plus en plus également, les analyses insistent sur les nombreux liens et interdépendances entre l'immobilier, le foncier, l'urbanisme et les politiques urbaines ou encore le système financier. Toutefois, l'essentiel de ces recherches se sont focalisées sur les métropoles négligeant ainsi les autres espaces. C'est donc dans une perspective davantage intégrative que s'inscrivent les réflexions que nous avons développées à partir des trois étages de Braudel.

Les idéaux-types proposés reposent sur une démarche de méta-synthèse à partir de plusieurs recherches menées en Suisse et de la littérature adoptant une approche socio-économique de l'immobilier. L'immobilier est un marché qui a ses propres caractéristiques. Il s'agit d'un bien qui comporte à la fois une valeur d'usage (consommation), d'échange (vente) et de rendement (profit). Sa «valeur» globale est largement contextuelle : elle dépend certes des caractéristiques de l'objet mais avant tout de sa localisation. Ici également, ce contexte renvoie à l'usage (comme la proximité d'un bassin d'emplois), mais aussi à la valeur d'investissement réelle ou financiarisée. Par exemple, l'immobilier d'une métropole possède une valeur sur les marchés financiers tandis que celui d'une ville moyenne n'en a généralement pas. Le cadre d'analyse développé tient compte de cette diversité territoriale mais également des imbrications existant au sein d'un même contexte. 
La typologie proposée ici ne constitue pas une théorie achevée de l'immobilier. Elle poursuit une autre ambition, celle de juxtaposer, comparer et articuler les différents objets, acteurs et institutions ainsi que les territoires constitutifs de l'immobilier. Cette approche relativement complète permet d'appréhender la dynamique générale du secteur et son rôle plus large dans l'économie et la société.

Alors que jusqu'à présent la majorité des travaux ont souligné le rôle des promoteurs, des constructeurs ou des investisseurs dans la dynamique du marché immobilier, la question de la rente urbaine, foncière et immobilière, est souvent considérée comme une « donnée », qui dépend d'une demande présente à un moment précis. Or, l'un des enjeux heuristiques majeurs consiste à déconstruire la valeur économique d'un objet immobilier. Cette valeur dépend d'un certain nombre de critères techniques, relatifs aux attentes d'utilité et de fonctionnalité des futurs utilisateurs par exemple. Elle dépend également de la rente commerciale escomptée, directement par les exploitants commerciaux, et indirectement via les loyers par les investisseurs et propriétaires. Elle dépend aussi des valeurs esthétiques et éthiques dans un contexte donné. Aujourd'hui en effet, les critères liés à l'esthétique et à la «beauté » du construit ne sont pas les mêmes qu'il y a dix ou trente ans. L'urbanisme et la qualité des constructions sont également de plus en plus calqués sur les attentes en matière de qualité de vie et de développement durable de la part des municipalités ou des habitants. La valeur économique d'un objet immobilier, en particulier celle de projets d'une certaine taille, est donc la somme pondérée de différentes valeurs sociales, qui peuvent faciliter ou empêcher sa réalisation. Par exemple, la qualité environnementale d'un projet peut être valorisée par les promoteurs. À l'opposé, les opposants à une opération immobilière s'appuient également sur certains critères éthiques ou sociaux, comme la nécessité de préserver l'espace public ou le patrimoine architectural d'un quartier. Ainsi, le « business model » des promoteurs immobiliers peut aujourd'hui s'avérer plus complexe en fonction des contextes territoriaux et du degré d'intervention des acteurs locaux tels que la municipalité et la société civile. 


\section{Références}

Aalbers M.B., (2008). The Financialization of Home and the Mortgage Market Crisis. Competition \& Change 12 (2), 148-66.

Aalbers M.B., (2009). The Sociology and Geography of Mortgage Markets: Reflections on the Financial Crisis. The International Journal of Urban and Regional Research 33, 281-290.

Agier M., (1999). L'invention de la ville - banlieues, townships, invasions et favelas. Éditions des Archives Contemporaines, Paris.

Aglietta M. et Orléan A., (1982). La violence de la monnaie. PUF, Paris.

Ascensão E., (2013). Following Architects and Engineers through Slums: The Technoscience of Slum Intervention in the Portuguese-Speaking Landscape. Análise Social 206, 154-180

Aveline-Dubach N., (2008). Immobilier : l'Asie, la bulle et la mondialisation. CNRS Éditions, Paris.

Ball M., (1998) Institutions in British Property Research: A Review. Urban Studies 35, 1501-1517.

Beauregard R., (1994). Capital Switching and the Built Environment: United States 1970-89. Environment and Planning A 26, 715-732.

Bolay J.-C., (2006). Slums and Urban Development: Questions on Society and Globalisation. The European Journal of Development Research 18 (2), 284-298.

Bourdieu P., Christin R., Bouhedja S. et Givry C., (1990). Un placement de père de famille. La maison individuelle : spécificité du produit et logique du champ de production. Actes de la recherche en sciences sociales $81-82,34-51$.

Braudel F., (1985). La dynamique du capitalisme. Flammarion, Paris.

Brenner N. and Theodore N., (2002). Cities and the Geographies of "Actually Existing Neoliberalism". Antipode 34, 349-379.

Butler T., (2007). Re-urbanizing London Docklands: Gentrification, Suburbanization or New Urbanism. International Journal of Urban and Regional Research 31, 759-781.

Camagni R., (1996). Principes et modèles de l'économie urbaine. Economica, Paris.

Charney I., (2001). Three Dimensions of Capital Switching within the Real Estate Sector: A Canadian Case Study. International Journal of Urban and Regional Research 25 (4), 740-58.

Corpataux J and Crevoisier O., (2005) Increased Capital Mobility/Liquidity and its Repercussions at Regional Level: Some Lessons from the Experiences of Switzerland and UK. European and Urban Regional Studies 4(12), 315-334.

Corpataux J., Crevoisier O. and Theurillat T., (2009). The Expansion of the Finance Industry and its Impact on the Economy: A Territorial Approach Based on Swiss Pension Funds. Economic Geography 85 (3), 313-334.

Crevoisier O., (2011). Beyond Territorial Innovation Models: The Pertinence of the Territorial Approach. Regional Studies 48 (3), 551-561.

Cuennet S., Favarger P. et Thalmann P., (2002). La politique du logement. Presses polytechniques et universitaires romandes, Lausanne.

David L. and Halbert L., (2013a) Constructing World-Class Cities: Hubs of Globalisation and High Finance. Dialogues in Urban and Regional Planning 5, 99-114.

David L and Halbert L., (2013b). Finance Capital, Actor-Network Theory and the Struggle over Calculative Agencies in the Business Property Market in Mexico City Metropolitan Region. Regional Studies 48 (3), 516-529.

Dubois O., (2002). Stratégies des acteurs de la construction résidentielle neuve et caractéristiques des espaces bâtis en Belgique. Belgeo 4, 319-332.

Ducan S. and Rowe A., (1993). Self-Provided Housing: The First World's Hidden Housing Arm. Urban Studies 30, 1331-1354.

Fainstein S., (1994 and 2001). The City Builders: Property Development in New York and London 1980-2000. University Press of Kansas, Lawrence.

Fainstein S., (2008). Mega-projects in New-York, London and Amsterdam, International Journal of Urban and Regional Research, 32 (4), 768-785. 
Finfgeld D. L., (2003). Metasynthesis: The State of the Art - So Far. Qualitative Health Research 13 (7), 893-904. Gerheuser F. W., (2004). Logement et conditions d'habitation : évolution de 1990 à 2000. OFS, Neuchâtel.

Guy S. and Henneberry J., (2000). Understanding Urban Development Processes: Integrating the Economic and the Social in Property Research. Urban Studies 37 (13), 2399-2416.

Hackworth J. and Smith N. (2001). The Changing Face of Gentrification. Tijdschrift voor Economische en Sociale Geografie 22, 464-477.

Hagermann L., Clark G. L. and Hebb T., (2007). Investment Intermediaries in Economic Development: Linking Public Pension Funds to Urban Revitalization. Community Development Investment Review 3, 45-65.

Haila A., (1991) Four Types of Investment in Land and Property. International Journal of Urban and Regional Research 15, 343-65.

Halbert L., (2004). The Intrametropolitain Decentralization of Business Services in the Paris Region: Patterns, Interpretation, Consequences. Economic Geography 80 (4), 381-405.

Halbert L. and Rouanet H., (2013). Filtering Risk Away: Global Finance Capital, Transcalar Territorial Networks and the (un)Making of City-Regions. Regional Studies 48 (3), 471-484.

Halleux J.-M., (2009). The Spatial Structuring of Interurban Housing Markets: Application to Building Sites Prepared for Self-Provided Housing. Environment and Planning A 41, 2143-2161.

Harvey D., (1978). The Urban Process Under Capitalism: A Framework for Analysis. International Journal of Urban and Regional Research 2 (1-4), 101-131.

Harvey D., (1985). The Urbanization of Capital. Basil Blackwell, Oxford.

Harvey D., (2003). The New Imperialism. Oxford University Press, Oxford.

Haumont N., (2001). Les pavillonnaires: étude psychosociologique d'un mode d'habitat. L'Harmattan, Paris.

Healey P., (1991). Models of the Development Process: A Review. Journal of Property Research 8, 219-238.

Healey P., (1992). An Institutional Model of the Development Process. Journal of Property Research 9, 33-44.

Healey P., (1998). Regulating Property Development and the Capacity of the Development Industry. Journal of Property Research 15, 211-227.

Healey P., (1999). Institutionalist Analysis, Communicative Planning and Shaping Places. Journal of Planning and Environment Research 19, 111-122.

Healey P., Davoudi S. and O'toole M., (2002). Property-Led Urban Regeneration: An Assessment In Healey et al. (Eds.), Rebuilding the City: Property-led Urban Regeneration, E. and F. N. Spon, London, 277-89.

Kemeny J., Kersloot J. and Thalmann P., (2005). Non-Profit Housing Influencing, Leading and Dominating the Unitary Rental Market: Three Case Studies. Housing Studies 20 (6), 855-872.

Keogh G. and D’Arcy E., (1999). Property Market Efficiency: An Institutional Economics Perspective. Urban Studies 36 (13), 2401-2414.

Lawson T., (1997). Economics and Reality. Routledge, London and New York.

Lees L., Slater T. and Wyly E., (2008). Gentrification. Routledge, New York.

Ley D., (2003). Artists, Aestheticisation and the Field of Gentrification. Urban Studies 40, 2527-2544.

Lizieri C., (2009). The towers of capital: office markets and international financial services, Wiley-Blackwell, Chichester.

Magalhaes C. S., (2002). Social Agents, the Provision of Buildings and Property Booms: The Case of Sao Paulo. International Journal of Urban and Regional Research 23 (3), 445-63.

Markowitz H., (1959). Portfolio Selection: Efficient Diversification of Investments. Wiley, Chichester.

Orléan A., (2011). L'empire de la valeur. Refonder l'économie. Le Seuil, Paris.

Poggio T., (2012). The Housing Pillar of the Mediterranean Welfare Regime. Relations between Home Ownership and other Dimensions of Welfare in Italy In Ronald R and Eslinga M (eds), Beyond Home Ownership. Housing, Welfare and Society, Routledge, London, 51-67.

Rérat P., (2012a). Gentrifiers and their housing choice. Characteristics of the households living in new developments in Swiss cities, Environment and Planning A, 44 (1), 221-236 
Rérat P., (2012b) The New Demographic Growth of Cities. Urban Studies 49 (5), 1107-1125

Rérat P., (2010). Habiter la ville. Éditions Alphil-Presses universitaires suisses, Neuchâtel.

Rérat P. and Lees L., (2011). Spatial Capital, Gentrification and Mobility: Evidence from Swiss Cities. Transactions of the Institute of British Geographers 36 (1), 126-142.

Rérat P., Moine A., Gertsch K. et Signoret P., (2011). La mobilité résidentielle transfrontalière dans l'Arc jurassien franco-suisse. Géo-Regards 4, 153-168.

Rérat P., Söderström O., Piguet E. and Besson R., (2010). From Urban Wastelands to New-Build Gentrification: The Case of Swiss Cities. Population, Space and Place 16 (5), 429-442.

Sassen S., (1991). The global city. Princeton University Press, Princeton.

Sassen S., (2010). Global Inter-City Networks and Commodity Chains: any Intersections? Global Networks 10 (1), 150-63.

Sayer A., (1992). Method in Social Science: a Realist Approach. Routledge, London and New York.

Smith N., (1979). Toward a Theory of Gentrification: a Back to the City Movement by Capital not People. Journal of the American Planning Association 45, 538-548.

Swyngedouw E., Moulaert F. and Rodriguez A., (2002). Neoliberal Urbanization in Europe: Large-Scale Urban Development Projects and the New Urban Policy. Antipode 34 (3), 547-582.

Theurillat T., (2010). La financiarisation de l'immobilier et la durabilité : le cas des investissements immobiliers des caisses de pension suisses. Éditions universitaires européennes, Saarbrücken .

Theurillat T., (2011a). La ville négociée : entre financiarisation et durabilité. Géographie, économie, société, 13 (3), 225-254.

Theurillat T., (2011b). Une approche territoriale de la financiarisation des régions et des villes et de la durabilité urbaine. Thèse de doctorat en Économie territoriale, Sciences humaines, Université de Neuchâtel.

Theurillat T. and Crevoisier O., (2012) The Sustainability of a Financialized Urban Megaproject: The Case of Sihlcity in Zurich. International Journal of Urban and Regional Research 37 (6), 2052-2073.

Theurillat T. and Crevoisier O., (2013). Sustainability and the Anchoring of Capital: Negotiations Surrounding Two Major Urban Projects in Switzerland. Regional Studies 48 (3), 501-515.

Theurillat T., Corpataux J. and Crevoisier O., (2010). Property Sector Financialization: The Case of Swiss Pension Funds (1992-2005). European and Planning Studies 18 (2), 189-212.

Thorne S., Jensen L., Kearney M. H., Noblit G. and Sandelowski M., (2004). Qualitative Metasynthesis: Reflections on Methodological Orientation and Ideological Agenda. Qualitative Health Research 14 (10), 1342-1365.

Torrance M. I., (2008). Forging local Governance? Urban Infrastructures as Networked Financial Products. International Journal of Urban and Regional Research 32 (1), 1-21.

Torrance M. I., (2009). The Rise of a Global Infrastructure Market through Relation Investing. Economic Geography 85 (1), 75-97.

Vorms C. (2009). Surproduction immobilière et difficultés d'accès au logement en Espagne, Études Foncières 138 (mars-avril), 21-26.

Walsh D. and Downe S., (2005). Meta-Synthesis Method for Qualitative Research: A Literature Review. Journal of Advanced Nursing 50 (2), 204-211.

Wheaton, W. (1999). Real estate “cycles": Some Fundamentals. Real Estate Economics 27 (2), 209-30.

Wood A., (2004). The Scalar Transformation of The U.S. Commercial Property-Development

Industry: a Cautionary Note on the Limits of Globalization. Economic Geography 80 (2), 119-140.

Zimmer L., (2006). Qualitative Meta-Synthesis: A Question of Dialoguing with Texts. Journal of Advanced Nursing 53 (3), 311-318.

Zukin S., (1982). Loft Living as "Historic Compromise" in the Urban Core: The New York Experience. International Journal of Urban and Regional Research 6, 256-267. 